\title{
Personalized Peripheral Information Awareness through Information Art
}

\author{
John Stasko, Todd Miller, Zachary Pousman, \\ Christopher Plaue, and Osman Ullah \\ College of Computing/GVU Center \\ Georgia Institute of Technology \\ Atlanta, GA 30332-0280 \\ stasko@cc.gatech.edu
}

\begin{abstract}
This article describes development of the concept of Information Art, a type of ambient or peripheral display involving user-specified electronic paintings in which resident objects change appearance and position to foster awareness of personally relevant information. Our approach differs from others, however, in emphasizing end-user control and flexibility in monitored information and its resultant representation. The article provides an overview of the system's capabilities and describes an initial pilot study in which displays were given to four people to use for an extended period of time. Reactions were quite favorable and the trial use provided suggestions for system improvements.
\end{abstract}

\section{Introduction}

Our lives are filled with information that influences what we do and how we feel. What world events are transpiring? How are my investments doing? Is our project at work nearing completion? What will the weather be like tomorrow? How much traffic will there be on the ride home? What's my child having for lunch at school?

As human beings, we naturally care about such questions as some may have important consequences for our lives while others may simply alter a small facet of our day-to-day experience. Maintaining awareness of such information helps to keep us more informed and presumably assists the multitude of decisions we make every day.

How do people stay aware of such information? Pervasive sources of information such as letters, flyers, newspapers, radio and television programs each have played important roles and they still do. Letters, flyers and newspapers, however, are predetermined and static - the information content is chosen by an author or editor and delivered as such. Conversely, many different radio and television programs exist, thus allowing more choices, but a person must be listening at the right time to acquire the desired information.

The Internet and the WWW now provide a new option for information discovery. Websites on almost any imaginable topic exist and can be opportunistically examined. Accessing a Website, however, frequently takes a few seconds if 
the site is known or possibly a few minutes if a search must be conducted. While that may not seem like a long time, the simple act of explicitly changing focus and the time it requires can be a significant disruption to a person's previous activity or train of thought.

The use of the periphery of human attention played a key role in Weiser's vision of ubiquitous computing as a calm computing resource - an attempt to mitigate these kinds of cognitive disruptions [24]. As he noted, "A calm technology will move easily from the periphery of our attention, to the center, and back." Furthermore, these types of calm technologies often can become aesthetically pleasing additions to a person's environment.

One form of ubiquitous computing, the ambient display, focuses on conveying low- to medium-priority information to people [25, 1]. An ambient display resides in the periphery of a person's attention. The display calmly changes state in some way to reflect changes in the underlying information it is representing. The designers of ambient displays typically stress display aesthetics, providing pleasant, attractive devices usually communicating a piece of information.

In [16], we introduced the InfoCanvas, a concept for a system that maintains some aspects of an ambient display, but provides greater access to more varied information sources. Such a system allows users to create electronic paintings in which objects in the scene represent information sources of interest, and the objects change appearance to reflect state changes in the information source they represent.

That earlier article was primarily an exploration of the concept of userdesigned information art. It also described an initial Web-based prototype system that sought to allow users to create their own virtual paintings beginning with a blank canvas upon which to add geometric objects using drawing tools such as pens, paintbrushes, fill markers, etc., or by choosing images from a palette of clip-art style objects. We eventually turned away from that approach, however, as potential users struggled, not knowing where to begin in creating their scenes. Also, potential users expressed that they did not feel artistically talented enough to create scenes that they would be comfortable displaying. Thus, the initial prototype we built was never used outside our research group, and it really only functioned as an exploration of the concept.

This article describes the details of a new, heavily-redesigned version of the InfoCanvas system that is currently being used by many different people now and has been the subject of two evaluation studies. Based upon our earlier experiences, we redesigned the system around the idea of "themes." A theme is a predefined, visually coherent scene that consists of a static background image with nearly all of usual scene objects removed, such as that of a tranquil meadow with only the grass, mountains, and sky shown. A theme also includes optional visual elements such as plants, animals, people, and inanimate objects that represent data of interest. Visual elements fit harmoniously within the background image, creating the illusion of a static piece of art. Elements also utilize a behavior from a set of predefined transformations in order to represent changes in the state of information being monitored. 
In the following sections, we describe related research work and then transition to describing the concepts employed in the system and its architecture and operations. The InfoCanvas breaks some existing notions of ambient and peripheral displays, and these differences will be highlighted. We also report on an informal pilot study in which the InfoCanvas was deployed to four people to use for an extended period of time. We describe reactions to the system and facts learned that are influencing its iterative design.

\section{Related Work}

Recently, a variety of systems have been developed to help communicate information to a person through channels that are not the primary focus of that person. These systems communicate important, but typically not vital, information in a calm manner using output devices ranging from computer monitors to tangible, real-world objects. The systems have been labeled with a variety of terms ranging from ambient displays to peripheral displays to notification systems.

Although no standard, accepted definitions of these terms exist, each has come to refer to slightly differing notions. Ambient displays typically communicate just one, or perhaps a few at the most, pieces of information and the aesthetics and visual appeal of the display is often paramount $[25,8,13]$. Peripheral displays refer to systems that are out of a person's primary focus of attention and may communicate one or more pieces of information $[19,14]$. Thus, peripheral displays would likely include ambient displays as a proper subset. Other types of displays such as scrolling tickers or animated news blurbs, however, would also be considered peripheral displays but likely not ambient displays.

Notification systems also deliver information in divided-attention situations in efficient and effective manners, but they more clearly including monitoring as a fundamental user task with the potential for people to react to important stimuli [15]. Ambient and peripheral displays more typically do not communicate critical, urgent information.

In addition to these types of systems, other awareness displays such as Web portals similarly may communicate many different pieces of information, but they typically do so as a person's primary focus of attention.

Examples of ambient displays, the first category described above, include Ambient Orb [2], Busmobile [13], Dangling String [24], Information Percolator[10], Lumitouch [4], Table Fountain [8], and Water Lamp and Pinwheels [5]. These systems often employ physical artifacts to represent information. The InfoCanvas differs from these systems in communicating more information in wider variety of representations.

Many peripheral displays, the second category listed above, use computer monitors to present information. They typically can communicate a greater variety of information in more flexible forms than the ambient display systems listed above, but do so by minimizing aesthetic considerations. Examples include KISS the Tram [12], Notification Collage [9], Scope [23], Sideshow [3], Tickertape [6], and What's Happening [26]. The InfoCanvas stresses aesthetics more than these 
system and provides a more abstract, symbolic representation of information. Another altogether different peripheral display approach is to use audio rather than visual presentations of information [17].

A few recent systems have used computer displays to convey information in artistic, attractive manners, and thus attempt to bridge the divide between information bandwidth and aesthetic considerations.

The Digital Family Portrait (DFP) [18] uses a computer display to simulate a picture frame and enclosed picture of a loved one. Iconic images in the display (chiefly the frame) represent data about the person's habits and well-being. The InfoCanvas and the DFP share general goals and presentation styles, but the DFP is a much more focused system on one particular domain. The InfoCanvas introduces a more general infrastructure, presumably one that could be used to implement a system just like the DFP.

The Kandinsky system [7] provides an artistic collage of images to represent email notes and news articles. Keywords in the text are used to retrieve particular related pictures that then are made into collages following different artistic styles. Kandinsky focuses on aesthetics as a primary goal, with information conveyance abilities considered almost an added bonus. As a result, information conveyance quality can vary tremendously from collage to collage. InfoCanvas, on the other hand, strongly emphasizes clarity of communication of the information state, even if the representation used is abstract and/or symbolic.

The Informative Artwork project $[21,11,22]$ is the most closely related research effort to ours. It uses LCD displays to produce subtly shifting representations based on well-known modern art pieces by artists such as Mondrian and Warhol. The two projects use the same semiotic approach with pictorial elements representing data, and both have a very similar goal: to facilitate people's peripheral awareness of information through attractive, artistic displays. Their focus is more on the aesthetics, however, and the data represented is usually predetermined and narrow, such as the current time, computer server traffic, or a set of weather forecasts. Each display they have shown has been custom-designed by the researchers themselves for a particular deployment. Our project differs in that we focus on end-user personalization and customization, and we explore the challenges involved in supporting people to design and specify their own information representations. Further, InfoCanvas displays typically consolidate more diverse information into a single display.

The SideShow system [3] is typically used to present very similar information as is done with the InfoCanvas and it supports user-customization as well, but that system uses a much more direct data representation including text and small standard iconic representations. Also, SideShow is primarily a peripheral computer desktop application that resides on one edge of a person's monitor, whereas the InfoCanvas is designed to be deployed in a more ecological location and blend into the surrounding decorations and furniture. 


\section{The InfoCanvas System}

Our objectives in developing the InfoCanvas were different than any of the existing peripheral display systems. We sought to provide an attractive display, one that would fit calmly and comfortably into a person's environment much as many others have, but we also wanted to provide end-users with the power and control to monitor information of personal relevance. The system should stress information communication (particularly of a moderate number, e.g., 5 to 15 , of different information items), but do so in a way that provides end-users with control and flexibility thus fostering their creative abilities. More specifically, the five objectives below have guided our efforts in building the current implementation of the InfoCanvas:

- Personalized - Rather than display some predetermined information source that different people may or may not be interested in, each InfoCanvas should be a highly customized information communicator for the person using it. The individual's particular personal information of interest should be presented.

- Flexible - A variety of information sources should be available for display, and should include the types of information that are the focus of peripheral awareness. If the data can be accessed via a Web page or an Internet information service, it should be available for use on an InfoCanvas.

- Consolidated - The system should support the presentation of a moderate number (5-15) of information sources and should consolidate their representation in one location, thus saving a person from having to check multiple devices or displays.

- Accurate - The system should accurately communicate the current state of monitored information. If for some reason an information source is not functional or not operating properly (which commonly occurs), this fact should be communicated to the user in a clear but non-distracting manner.

- Appealing - Simply put, the system should be fun to use and should be an aesthetically pleasing addition to a person's environment.

The basic premise of the InfoCanvas is to allow a user to create a visual scene that serves as an abstract representation of information that is relevant to them. The result is an aesthetically pleasing "picture" that appears to be nothing more than an artistic rendering of a scene, such as a beach or cityscape. The picture is presented on a LCD display and subtly changes to reflect updates in the information being tracked. The InfoCanvas can be displayed in a manner like that of a painting or calendar on a wall, or even a picture on a desk. The goal is to blend into the physical environment of the user, thus providing a type of "virtual painting" or an "electronic illustration." Figure 1 shows an example InfoCanvas mounted on a wall in an office.

As mentioned earlier, an initial prototype InfoCanvas that provided drawing tools (pen, paintbrush, fill, etc.) and a small palette of clip-art icons for display design was developed [16], but creating views was too open-ended and difficult. This experience led us to redesign the system around the idea of themes. A 


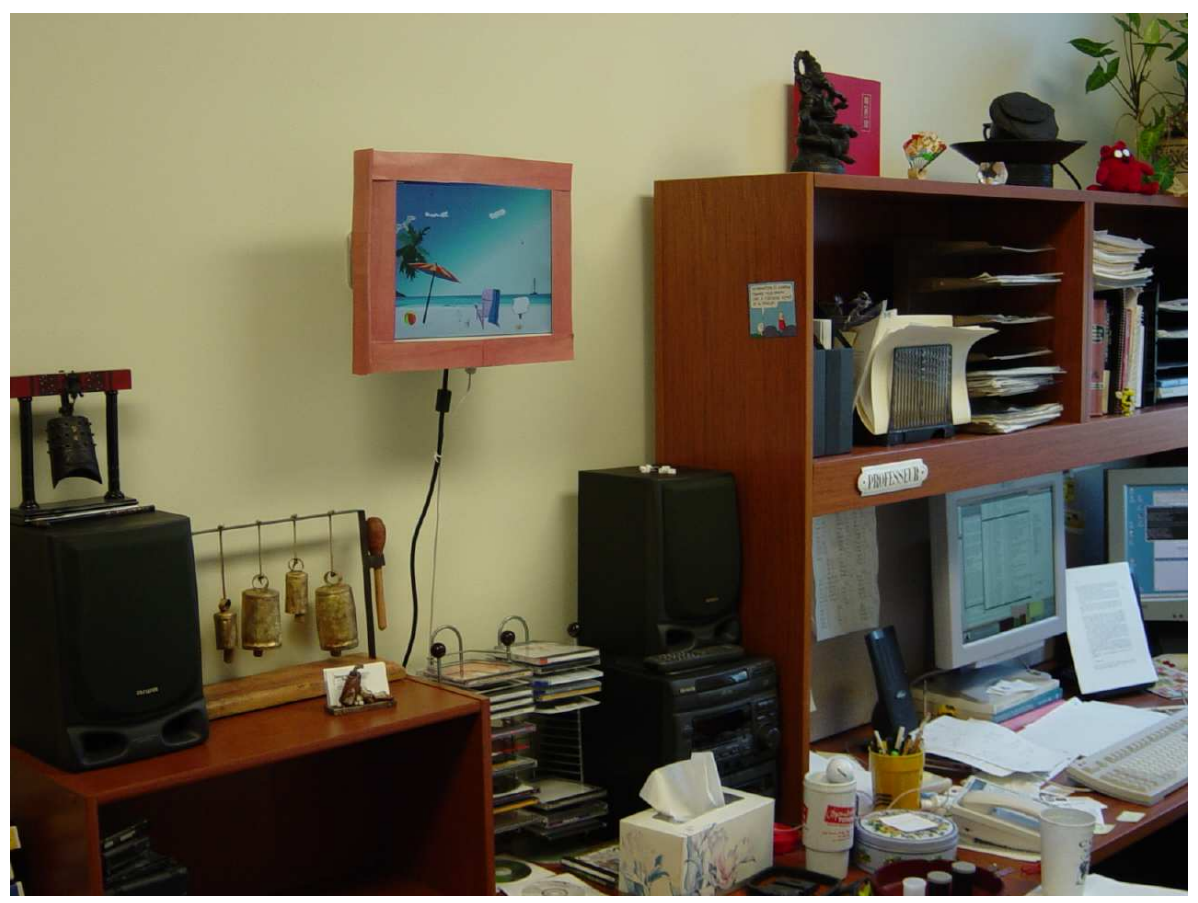

Fig. 1. An example installation of the InfoCanvas mounted on a wall like a picture.

theme consists of a static background image with most of the usual objects removed, such as that of a beach with only the sand, the water, and the sky pictured. A theme also includes many optional visual elements that can be placed on it such as seagulls, palm trees, sailboats, crabs, and blankets for the beach theme. Elements are designed to fit both stylistically and thematically with the background image, thus mimicking a static illustration, picture, or painting. Many different visual elements can be in a display, thus promoting our objective of consolidation in the awareness display.

An element added to a view represents a specific piece of information being "watched" by the user. A mapping between the state of the information and the visual presentation of the element is constructed. As the information being monitored changes, the visual element representing it also updates its rendering to communicate that change. We call these changes to elements transformations. For example, an InfoCanvas could include a flower element that appears whenever a person has received an email from their spouse within the last hour. A bird in an InfoCanvas could represent the daily change of the stock market, with the bird flying higher when the market is up and lower when the market is down. We use the term scene to refer to an "active" theme that is representing specific data with various mapping transformations applied to it.

The following set of transformations are included in the InfoCanvas: 
- slider - An element moves along a straight line to represent different values of an information source. The two endpoints of the line represent minimum and maximum values of the information. Example: A crab moves horizontally along the beach to represent the current airfare between two specific cities.

- swapper - A set of visual elements is available, and a specific one is displayed depending on the state of the information source. Example: The bathing suit color of a person on the beach changes to represent the traffic speed on a particular road (green-fast, yellow-moderate, red-slow).

- appearance - A visual element appears when a particular condition is true and is not shown when the condition is false. Example: A towel element appears on a chair on the beach when a particular Web page includes a specified keyword.

- scaler - An element changes size to represent different values of an information source. Example: A sailboat grows and shrinks to represent the forecasted temperature.

- population - Repeated copies of an element are displayed to represent a value of an information source. Example: A drink glass appears on the beach for each ten unread emails in a user's queue.

- display - An image or a text string taken directly from an information source is displayed on an InfoCanvas. Example: A lead image from a news Web site is displayed on a billboard in a scene.

Note that the designer of an InfoCanvas has the freedom to specify mappings that are either more concrete/direct or more abstract/symbolic. For instance, the slider element used to represent a best airfare could be an airplane in the sky or it could be a crab on the beach. Similarly, tomorrow's weather forecast may be represented by an image swap putting different weather icons (sun, clouds, lightning, snow) in the sky or it could be an image swap placing different types of cars on a city street. This capability supports the goals of providing mappings that are both flexible and personalized.

Figure 2 illustrates examples from the wide variety of InfoCanvas themes created by different members of our research group. Themes range from collections of clip-art and hand-drawn objects to photo-realistic scenes as well as artistic Japanese watercolor designs. In the photo-realistic city street theme (at upper right), the trolley car and the bicyclist are sliders; the police car roof lights and a street lamp are image appearance elements (on/off); various cars are image swaps (different car or different color); people on the sidewalk provide a population element; and the awning above a store changes color in an image swap.

Although the freedom to flexibly define a scene exists, we also promote certain conventions to follow in theme design to ensure coherent scenes. For instance, in a display transformation, image or text should only be displayed in a context and a manner fitting to the theme, thus ensuring visual continuity. Thus, rather than an image arbitrarily appearing in a scene, it should only appear in locations that a picture typically would be seen, such as on a billboard, on a sign, or on a television screen. Similarly, a text string could appear as a banner being pulled by an airplane or on a note lying on a beach. 




Fig. 2. An assortment of different InfoCanvas themes that have been built.

Calling these scenes "art" may be a bit presumptuous as they reflect more of a clip-art, decorative style. But for a picture to have many different objects that can be moved, altered, or scaled and still look properly integrated, displays likely must take this style. Moving and modifying pieces of great paintings (at least ones other than abstract art) would surely erode their visual appeal.

Internet-based information resources such as Websites may go offline or become temporarily unavailable. As a result, information provided will not be available for presentation on the InfoCanvas. To signify such an event, the data's visual element on the InfoCanvas becomes semi-transparent. This promotes the accuracy objective by communicating the data acquisition problem to the viewer, but does so in a more subtle, non-distracting manner that does not destroy the visual continuity of the scene. 
The InfoCanvas is written in Java, containing modules to monitor information sources, display scenes, and update visual elements as needed. Accompanying the system is a growing collection of themes, each residing in its own folder and providing a background image and a collection of visual element images. The InfoCanvas takes as input a configuration file specifying the visual theme to be presented, the data to be monitored, and the transformations from data to representation. The configuration file is in XML format and is described in more detail later in this section. Users simply create and modify the configuration file with a text editor. The InfoCanvas then uses the configuration file to construct an initial scene and thereafter refreshes the display at preset intervals. Once the program is started, the no further user intervention is required.

Using a comprehensive "driver" configuration file allows the system to be used in a variety of ways. First, users can take pre-existing scenes with all the elements and transformations specified, but simply substitute in their own personal information sources to drive the transformations. Second, users can modify the transformations in a scene, perhaps even adding new visual elements and information mappings. Finally, the truly ambitious designer can create a theme from scratch beginning with a background image and adding as many visual elements as desired. Presently, a GUI interface is being developed to simplify the transformation specification process and make creating new configuration files easier. Users will interactively position and scale visual elements via menus and control palettes.

Each InfoCanvas scene is stored in the form of an XML file whose format is strictly specified by a Document Type Definition (DTD). The file specifies the theme: name, canvas size, working folder for images, the background image, and a list of visual elements. Visual elements can either be static or active. Static visual elements are images that do not change; they are simply decorations for the scene and are separated from the background to provide more flexibility in scene design. Active visual elements are objects that represent information using one of the six transformations listed earlier (slider, swapper, appearance, scaler, population and display). An active visual element consists of an image or a set of images, the data it represents, and a specification of how the data will transform the image(s).

An example of an active visual element with a slider transformation is shown below. (The XML format uses the term "object" for a visual elements.) This transformation uses a 40-pixel-wide by 22-pixel-high seagull that moves from coordinates $(250,370)$ to $(250,0)$ to represent temperature values between 0 and 50 degrees in the geographic area corresponding to postal code 17837.

Note the use of the term harvester in the specification. Harvesters are system objects that collect information. Presently, a wide variety of harvester classes exist, gathering data for weather, traffic, email, stock market data, and Web page text strings and images. The paramname argument of a harvester identifies the specific aspect of the information class to be queried. For example, the weather harvester includes categories for the current temperature, current conditions, tomorrow's forecasted high and low temperatures, tomorrow's forecasted con- 
ditions, etc. Harvesters also use data specific to the harvester type such as the zip code of the geographic region being watched in this example. At a specified interval, a harvester gathers all of its potential data and stores it in a hash table. To prevent harvesters from querying sources more than necessary, each harvester supports a specific harvest interval. For example, a stock harvester will have a shorter harvest interval than a weather harvester.

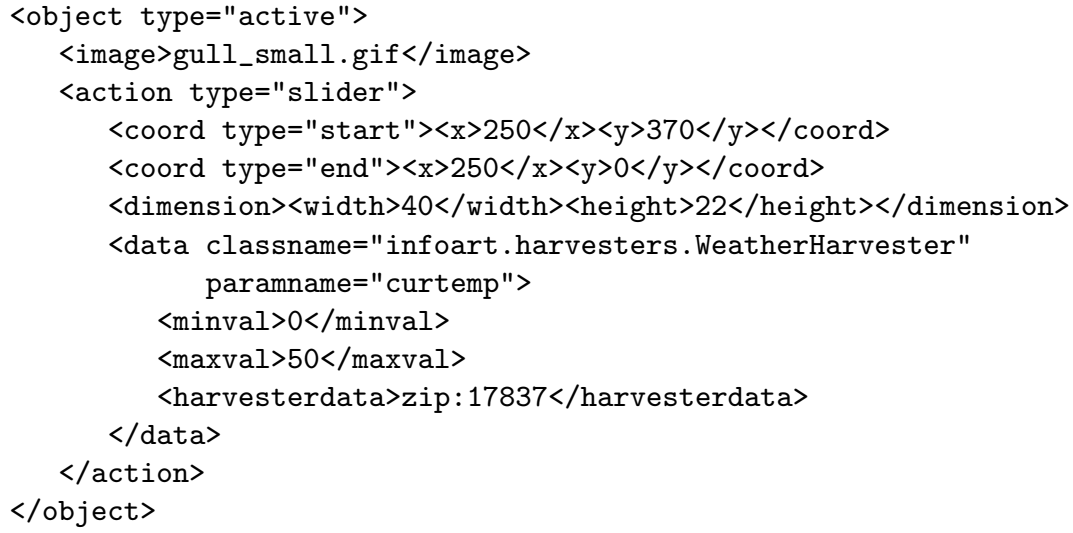

Once the InfoCanvas reads the XML configuration file, an internal data structure is created. This data structure consists of images (visual elements), harvesters, and the transformations that link images and harvesters together by specifying the data range for the information as well as how the data from the harvester will modify the image. Once the data structure is built, the InfoCanvas polls each harvester at a regular interval by calling its harvest() method, then extracting the desired information from the updated hash tables. If a harvester is polled before its harvest interval has passed, it will return the same data as the last query. This cycle of polling, harvesting, and updating the display continues until the program is terminated.

\section{Evaluation}

Evaluating any form of ubiquitous computing application or service is challenging, and that certainly holds true for peripheral displays. Mankoff et al. observe that most ambient displays have not been evaluated at all, and little is known about what makes one display more effective than another [13]. To address this problem, they developed and refined a set of principles for use in guiding a discount heuristic evaluation of an ambient display. This technique is aimed at evaluating a system in its formative stages by analyzing the system with respect to various heuristics. These criteria thus can serve as initial evaluation metrics with which to discuss and consider the InfoCanvas.

First, on heuristics such as "Useful and relevant information," "Peripherality of display," "Aesthetic and pleasing design," and "Match between design of ambient display and environment," the InfoCanvas would, we believe, score well. 
Each of these is fundamental to the design of the system. The "Sufficient information design" heuristic is about whether too much or too little information is displayed. The InfoCanvas allows users to decide how much and what type of information to convey, therefore it presumably would rate high on that heuristic as well.

Perhaps most interesting are those heuristics on which the InfoCanvas might be rated low. The first stresses the creation of a "Consistent and Intuitive Mapping." The InfoCanvas, however, may use abstract mappings, some of which are not intuitive at all. Another heuristic encourages designers to provide an "Easy transition to more in-depth information." The InfoCanvas intentionally did not allow users to drill down for more information because we wanted to promote a calm, passive interface. (Note that this design decision was questioned and will be discussed later in this section.) Finally, the heuristic "Visibility of State" means that a display should make system states noticeable and state-to-state transitions easily perceptible. The InfoCanvas' use of semi-transparent visual images follows this heuristic as does the general emphasis of clarity in representation state, but we also promote "change-blind" displays in which image updates are relatively difficult to notice and no attention-grabbing visual effects such as animation are employed.

All three of those heuristics would probably not be judged by expert evaluators to be fully realized in the InfoCanvas, but this is not an omission on our part. Rather, these characteristics were consciously designed features of the system. We posit that there are other systems, like ours, that by design reject a heuristic to achieve system goals. In this way, the heuristics can be viewed not just as assisting the evaluation of ambient and peripheral display systems, but also in their design.

Our own to-date evaluations of the InfoCanvas have focused on its objective ability to convey information and the subjective impressions of people with respect to its usefulness and appeal. We also wanted to observe just how people would use the system, the representations they would select, and any problems or unanticipated findings that would arise.

To evaluate its objective information communication capabilities, we conducted an experiment comparing the InfoCanvas to a Web portal-style display and a text display, examining each display's ability to transmit information to people at a glance [20]. We encoded ten different types of information (news, weather, traffic, etc.) together onto each the three different display types respectively, and then we showed instances of each display to experiment participants for eight seconds. Each instance encoded a different set of ten values. The individual then had to recall the value or state of the information sources as presented in the display. Participants in the experiment recalled a statistically significantly higher number of information values per display with the InfoCanvas than with the other two display types. Because the information source-to-visual element mappings were defined by us and not the participants, the result is particularly meaningful in showing that people can learn the types of abstract mappings in an 
InfoCanvas quickly, and they are able to comprehend, translate, and understand the visual transformations relatively well.

To evaluate the perceived usefulness and aesthetics of InfoCanvas displays, a longitudinal study of actual system use was conducted. Four people were recruited to create their own version of the InfoCanvas and use it in their offices for a period of about two months. We considered this an initial trial evaluation effort and our goals were relatively modest - to gather initial impressions about people's impressions of the system and to gain suggestions for changes or improvements to feed our iterative design process. We were not evaluating their ability to create or configure a display.

A second video card and LCD monitor were purchased to attach to a participant's primary computer. This choice was made for a number of technical, financial, and practical reasons. This decision likely would affect the study in that participants could potentially utilize the second monitor as additional workspace, placing application windows on top of their canvas display. While this could not be easily prevented, participants were instructed that the monitor should be solely for use as an InfoCanvas display.

\subsection{Study Methodology}

At a first meeting with each participant, we asked them to list information that they currently check semi-regularly, such as email, weather forecasts, or news headlines. The interviewer probed for more details on each item, such as the specific Web site(s) visited, what time(s) throughout the day the information is examined, what information in particular is of the most interest, their motivations for checking, and how they might respond to the information. The interviews lasted approximately thirty minutes.

Within one to two weeks following the initial interview, we performed a more intensive design interview session. At the start of the interview, the information currently monitored by the participant was reviewed, and the person was able to make further clarification or additions.

After the review, we explained to the participant the concept of the InfoCanvas in detail. We summarized how the display will function in their environment and the means by which information can be graphically represented. The study used a set of six themes from which a participant could select their own personal scene. Each theme had at least twenty visual elements that could be used for representing data.

In order to provide a natural, creative process for participants to design their InfoCanvas, we decided to use paper prototyping in the design sessions. This hands-on approach involved giving participants paper cut-outs of images that could be moved around the background image. Participants were told that the size of an element could easily be changed to fit better in the scene.

When a participant selected a theme for use in their canvas, we presented the individual with a paper copy of the theme's background image and the paper cut-outs of visual elements available for use. We would then suggest an item to monitor from the participant's list of information and provide any needed 
assistance as they created a mapping of that data to a visual element. The mapping was made by placing a cut-out image onto the paper background of their canvas and verbally describing how it would function. This process was repeated for each piece of information to be represented. At the end of the interview, we asked the participant to point out each element and explain what it represented and how it functioned. This action clarified the final mappings as well as tested the participant's ability to remember their design. The entire design interview lasted about an hour.

Within a few days of the design interview, we implemented a functional version of the participant's design and installed it in their office. Participants then were contacted weekly and asked a short series of questions to gauge usage of their InfoCanvas. The questions examined how frequently they looked at their InfoCanvas, what information was most useful, if they had any difficulty remembering or interpreting the visual mappings, and whether any technical difficulties had arisen over the past week.

During the weekly interviews, we encouraged participants to suggest additions or changes to their design, some of which we subsequently implemented depending on the feasibility. This provided the participants added control, allowing them to keep their InfoCanvas useful by updating the representation and adjusting inadequate representations.

After roughly two months had passed, the InfoCanvas was removed from the environment and a final interview was conducted. The participant was asked to comment on their overall experience with the system, including their likes and dislikes, and if the system had any effect on their daily routine.

Our study involved four local people: two faculty members in our own department and two administrators at a nearby university. Two participants chose an aquarium theme, one selected a beach theme, and another selected an office view. (Only the beach theme from the set shown in Figure 2 had been created at the time of the study.) Figure 3 shows where the InfoCanvas was positioned for each person and a sample view of their theme while it was running.

\subsection{Study Findings}

\section{Utility}

All participants stated that they enjoyed having the InfoCanvas display and thought that it was a useful means to portray information. Participant A, before receiving her InfoCanvas, questioned "How is this different from a My Yahoo page? Why would you need a separate screen, a separate application?" She wondered what the advantage would be and thought that a direct, textual representation of information would make more sense. However, after having used the InfoCanvas for an extended period of time she commented, "[The InfoCanvas] has a different feel. It is more private, because the casual observer will not know the meaning of these icons. They won't know you just got email from [your spouse]. In that regard it was unique and different from anything that I have on my computer." 


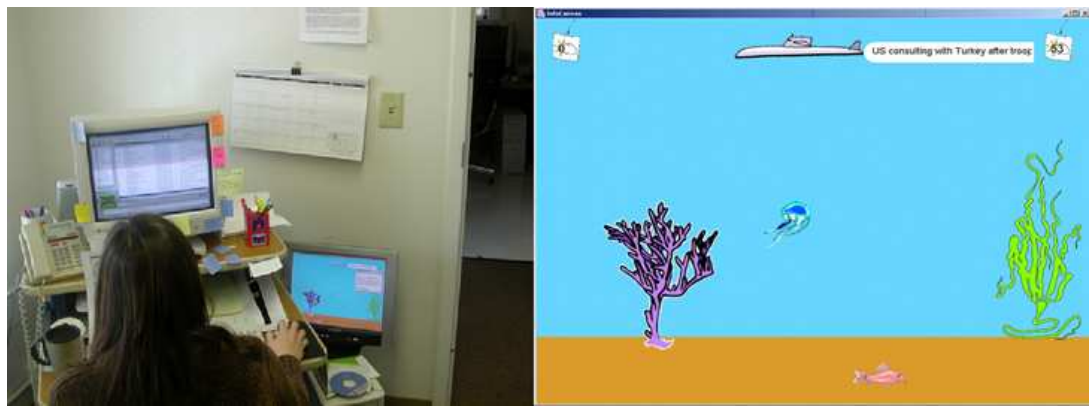

Participant A
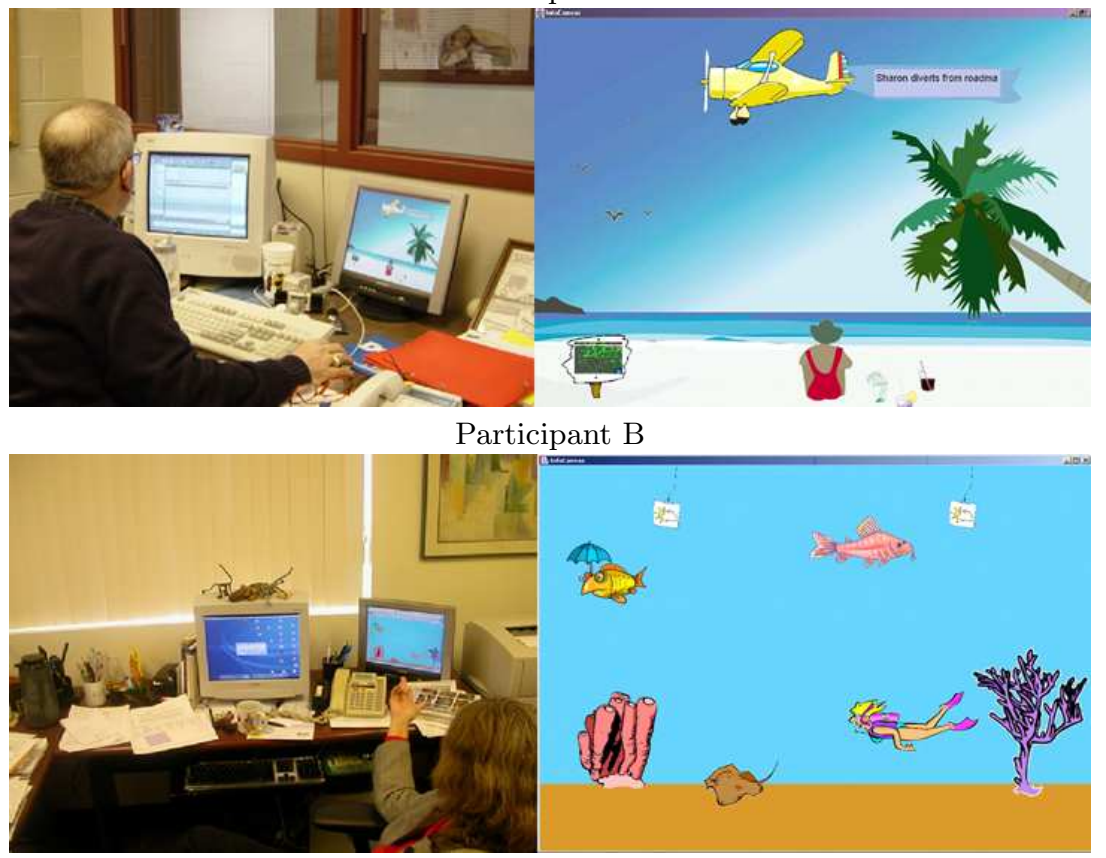

Participant C

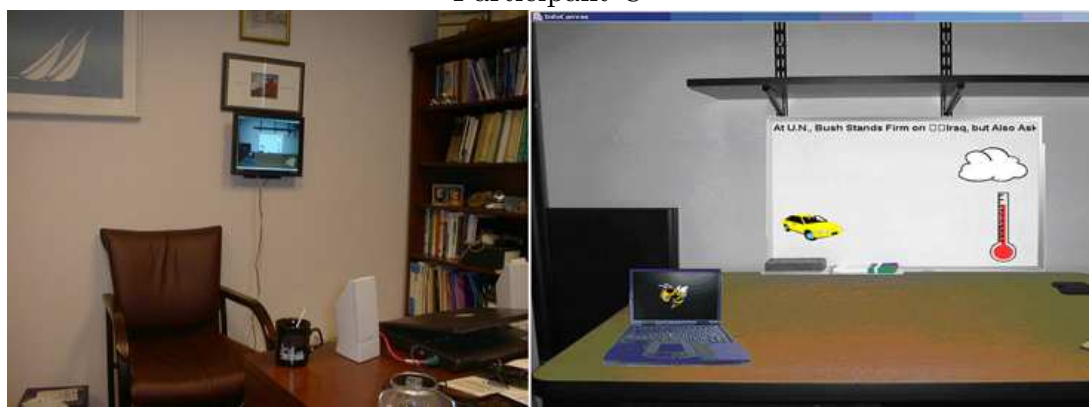

Participant D

Fig. 3. Shown are the deployment configurations and a sample view from each of the four study participants using the InfoCanvas. 
All participants reported that they would look at their InfoCanvas frequently throughout a day. Participant B stated, "I check it when I first come in, in between tasks, and whenever I come back from a meeting. It's situated right where my eyes go when I sit back to think for a second, so I'm always noticing it." In addition, no participant reported their canvas as distracting or felt that it interrupted their normal workflow.

\section{Data Representations}

We noted certain trends in the manner that each type of data was represented by the participants. Boolean data, such as whether or not a Web page has been updated, was represented by appearance transformations or by an image swapper with two visual elements. These transformations were apparently the most intuitive mappings, even though others are equally feasible, such as an image slider moving from one side of the display to the other.

Ordinal data was represented by an image swapper or by a population transformation. For example, participant C's coral changed color according to the temperature, using blue for below 50, hot pink for above 80 degrees, and orange for values in between. Traffic was represented by the number of boats sailing on participant B's canvas, with more boats signifying more congestion.

Continuous data was represented by almost every means possible, including sliders, swappers, or direct displays (value shown as digits). The method chosen appeared to correlate with how important the data was to the person and thus it dictated the precision of the representation. For example, participant C's scene utilized an image swap with a happy or sad fish to represent Coca-Cola's daily stock price movement. This mapping conveyed very little about the actual value of the stock, as a gain of half a point would show the same happy fish as a gain of ten points. However, she stated "I really love the happy and sad fish! They're just so cute!" and was satisfied with the level of awareness provided. However, participant D wanted to monitor the three major stock market indices precisely and chose to have the current day's change shown directly as numeric values written on a notepad.

The textual data monitored was mostly limited to news headlines or weather forecasts and was commonly represented in a fairly direct or literal mapping using a swapper or a text display. The natural weather images of the sun, clouds, and rain were used to represent forecasts, and news headlines were always written out textually. One exception was the monitoring for the appearance of stories about evolution on a newspaper Website by participant $\mathrm{C}$, which was represented by a stingray appearing.

Not all themes had elements that lent themselves to intuitive mappings for all of a participant's data. However, participants were innovative in layering elements and creating abstract representations that allowed them to overcome the limitations of the pre-designed themes and paper cut-outs. For example, the stingray used by participant $\mathrm{C}$ to represent news stories about evolution does not have a strong visual correlation to evolution, but she had been stung by one in the past and her personal experience helped her to make the connection between the image and the data monitored. Participant A wanted to know the exact high 
temperature forecast, but was using the aquarium theme. She decided to layer the numerical value on top of a little sign with the forecasted condition dangling in the water from a hook and line. Thus the numerical value was contained within an element and maintained the visual continuity of the theme. It worked so well that when participant $\mathrm{C}$, who worked at the same institution, happened to see it, she immediately requested that we add it to her scene.

Many of the representations chosen by the participants were not intuitive. As mentioned briefly earlier, this finding potentially conflicts with the Mankoff [13] ambient display evaluation heuristic stating that a display should use intuitive representations, because abstract transformations may require too much cognitive processing by the user. Our to-date use of the InfoCanvas has illustrated that people enjoy the highly symbolic mappings it facilitates. The system even generates its own lingo, with expressions such as "The crab's really to the left today - The market is plummeting," becoming commonplace. If one interprets the intuitive heuristic as simply meaning that states of the display are perceptually easy to recognize, then the InfoCanvas matches better.

\section{Ability to Remember and Interpret}

No participant reported any difficulty in remembering the mappings that they had created between data and visual elements. Participants also reported that they had no problem understanding the information portrayed on the InfoCanvas. However, two participants did report some difficulty in interpreting the value represented by sliders. Participant A's jellyfish moved horizontally along a path to represent the daily performance of a mutual fund. While she could tell whether the fund was up or down, she found that it was difficult to translate that into a more meaningful number. Participant B had a seagull that represented the current temperature based on the bird's height in the sky. The range was so large (30 to 90 degrees) that it was difficult to tell the difference between small but important differences in temperature. The other movement based mappings employed by these participants were not problematic.

\section{Interaction with the Display}

The desire to use the InfoCanvas as an interactive data exploration tool to investigate information was expressed by all participants. This was motivated by a desire to check information that is represented on their canvas, or by noticing that an interesting change in a representation when glancing at their display.

Participants stated that they wanted to be able to mouse over a particular item to receive more information, and furthermore, to be able to click on the item to launch a Web browser or other program that would allow them to quickly get the full details behind the visual element. In response to the first request, we added a mouse-over tool-tip capability to the system. When the mouse cursor moves over a visual element, a tool-tip shows a simple detail, such as the number of new emails or the current temperature. This was one example where feedback from the study has directly influenced system functionality.

We were slightly uneasy about adding this tool-tip capability because it seemed to violate one of our primary goals of making the InfoCanvas be a calm 
information communication conduit not requiring explicit user interaction. We wondered if this capability was requested because three of the four participants had their monitors on a nearby desk rather than in a more peripheral location such as being mounted on a wall. Interview feedback indicated to us that the participants still thought strongly of the display as a computer monitor, not as a separate stand-alone service.

This initial study provided us with some initial feedback about actual use of the InfoCanvas, but a more careful, comprehensive study is still needed. Presently, we are preparing a more in-depth, longitudinal study that will involve more users and will include both the subjective, qualitative analysis as present in this study and more highly analytical comparisons of people's use and perceptions of the system. In the new study, the InfoCanvas will be hung on a wall or on a shelf more appropriately, and we will see if user views, such as the desire for interaction, still occur.

\section{Summary}

We have described the concept of Information Art and how it is manifested through the InfoCanvas system. While the system stresses calm, aesthetically pleasing communication of information like other peripheral displays, it differs in providing a user with more control over the information being monitored and the representation of that information. We described the use of themes with visual elements that undergo transformations as a way to assist users to construct attractive and illuminating scenes. We also presented results from an initial study of extended use of the system.

Although current use of the InfoCanvas is primarily as a picture on a wall or desk, one can imagine any number of other possible uses. For instance, an InfoCanvas scene could be shown as a computer's screen-saver or displayed on an extra monitor in a multi-monitor display. ${ }^{1}$ Similarly, as televisions and computers become more integrated, an InfoCanvas could be shown on a television when it is turned off. Further, it is possible to imagine simple InfoCanvas themes being used as the "off" displays for small devices such as PDAs and watches.

\section{Acknowledgments}

This research has been supported in part by a grant from the National Science Foundation, IIS-0118685. Many students have assisted with the project including Julie Hoffman, David Browning, Alex Drake, Nena Hy, Toni Pashley, Shannon Bauman, and Jehan Moghazy.

\footnotetext{
${ }^{1}$ Here, the display should not be a CRT because of the pixel burn-in issue with relatively static scenes such as those in the InfoCanvas.
} 


\section{References}

1. Abowd, G., et al.: The Human Experience. Pervasive Computing 1 (2002) 48-57

2. Ambient Orb. Ambient Devices Inc. www. ambientdevices.com

3. Cadiz, J. J., et al.: Designing and deploying an information awareness interface. Proc. CSCW (2002) 314-323

4. Chang, A., et al.: Lumitouch: An emotional communication device. Extended Abstracts of CHI (2001) 371-372

5. Dahley, A., et al.: Water Lamp and Pinwheels: Ambient projection of digital information into architectural space. CHI Extended Abstracts (1998) 269-270

6. Fitzpatrick, G., et al.: Tickertape: Awareness in a Single Line. Proc. CHI (1998) 281-282

7. Fogarty, J., et al.: Aesthetic information collages: generating decorative displays that contain information. Proc. UIST (2001) 141-150

8. Gellersen, H. W., et al.: Ambient display media for peripheral information display. Personal Technologies 3 (1999) 199-208

9. Greenberg, S., Rounding, M.: The Notification Collage: Posting information to public and personal Displays. Proc. CHI (2000) 515-521

10. Heiner, J. M., et al.: The Information Percolator: Ambient information display in a decorative object. Proc. UIST (1999) 141-148

11. Holmquist, L. E., Skog, T.: Informative art: Information visualization in everyday environments. Proc. Graphite (2003) 229-235

12. Lunde, T., Larsen, A.: KISS the Tram: Exploring the PDA as support for everyday activities. Proc. Ubicomp (2001) 232-239

13. Mankoff, J., et al.: Heuristic evaluation of ambient displays. Proc. CHI (2003) $169-176$

14. Matthews, T., et al.: A Peripheral Display Toolkit. Intel Research Berkeley Tech. Report IRB-TR-03-018 (2003)

15. McCrickard, D. S., et al.: A Model for Notification Systems Evaluation-Assessing User Goals for Multitasking Activity. ACM Trans. on Computer-Human Interaction 10 (2003) 312-338

16. Miller, T., Stasko, J.: Artistically conveying information with InfoCanvas. Proc. AVI (2002) 43-50

17. Mynatt, E., et al.: Designing audio aura. Proc. CHI (1998) 566-573

18. Mynatt, E., et al.: Digital family portraits: Providing peace of mind for extended family members. Proc. CHI (2001) 333-340

19. Pedersen, E. R., Sokoler, T.: AROMA: abstract representation of presence supporting mutual awareness. Proc. CHI (1997) 51-58

20. Plaue, C., et al.: Is a Picture Worth a Thousand Words? An Evaluation of Information Awareness Displays. Proc. Graphics Interface (2004) 117-126

21. Redstrom, J., et al. Informative Art: Using Amplified Artworks as Information Displays. Proc. DARE (2000) 103-114

22. Skog, T., et al.: Between aesthetics and utility: Designing ambient information visualizations. Proc. Information Visualization (2003) 233-240

23. van Dantzich, M., et al.: Scope: Providing awareness of multiple notifications at a glance. Proc. AVI (2002) 267-281

24. Weiser, M., Brown, J. S.: Designing calm technology. Power Grid Journal 1 (1996)

25. Wisneski, C., et al.: Ambient Displays: Turning Architectural Space into an Interface between People and Digital Information. Proc. CoBuild (1998) 22-32

26. Zhao, Q. A., Stasko, J. T.: What's Happening?: Promoting Community Awareness Through Opportunistic Peripheral Interfaces. Proc. AVI (2002) 69-74 